\title{
Laparoscopic Single Figure of Eight Suturing Omentopexy for the Treatment of a Perforated Duodenal Ulcer
}

\author{
Jung Jun Yoon, M.D., Hyung Ook Kim, M.D., Ph.D., Kyung Uk Jung, M.D., Sung Ryol Lee, M.D., Ph.D. \\ Department of Surgery, Kangbuk Samsung Hospital, Sungkyunkwan University School of Medicine, Seoul, Korea
}

Purpose: Safe and effective surgical treatment of peptic ulcer perforations is fundamental to achieve favorable outcomes. We present laparoscopic single figure of eight suturing omentopexy for perforated duodenal ulcer and review associated clinical outcomes. This is a new formulaic surgical technique for laparoscopic omentopexy.

Methods: Laparoscopic single figure of eight suturing omentopexies for perforated duodenal ulcer were completed in 15 consecutive patients between April 2008 and November 2017 at Kangbuk Samsung Hospital, Sungkyunkwan University School of Medicine, Seoul, Korea. Using prospectively collected data, we performed an observational study on an intention-to-treat basis.

Results: The mean age of the 15 patients who underwent laparoscopic repair was $41.7 \pm 13.9$ years. The perforation site was the anterior duodenal bulb in all patients. The median perforation size was 5 $\mathrm{mm}$ (range: 3 8 mm). The mean operation time was $66.7 \pm 19.6$ minutes. There was no evidence of leakage from the omentopexy site clinically or in the postoperative upper gastrointestinal series. One patient $(6.7 \%)$ experienced the postoperative complication of pneumothorax. There were no cases of postoperative mortality or reoperation within 30 days after surgery. The median time to tolerance of regular diet was 6 (range: 4 9) days. The median postoperative hospital stay was 7 days (range: 5 11 days).

Conclusion: Laparoscopic single figure of eight suturing omentopexy can be a viable option in the surgical management of perforated duodenal ulcer in selected patients without surgical risk factors. Laparoscopic single figure of eight suturing omentopexy is safe and easy to perform, and may therefore reduce operation time.

Keywords: Peptic ulcer perforation, Duodenal ulcer, Laparoscopy, Omentopexy

This is an Open Access article distributed under the terms of the Creative Commons Attribution Non-Commercial License (http:// creativecommons.org/licenses/by-nc/4.0/) which permits unrestricted non-commercial use, distribution, and reproduction in any medium, provided the original work is properly cited.
Received July 4, 2018

Revised 1st July 24, 2018

2nd July 26, 2018

Accepted August 1, 2018

Corresponding author

Hyung Ook Kim

Department of Surgery, Kangbuk

Samsung Hospital, Sungkyunkwan

University School of Medicine, 29

Saemunan-ro, Jongno-gu, Seoul

03181, Korea

Tel: +82-2-2001-8550

Fax: +82-2-2001-2131

E-mail: ho115.kim@samsung.com ORCID:

http://orcid.org/0000-0003-0603-8766

This paper was presented with poster at the meeting of the 42th Korean Society of Endoscopic and Laparoscopic Surgeons (KSELS) \& 8th International Symposium, Jeju, Korea, April 20-21, 2018.

Copyright (C) 2019 The Journal of Minimally Invasive Surgery. All rights reserved.

\section{INTRODUCTION}

Perforated peptic ulcer is one of the most serious complications of peptic ulcer disease, and is characterized by mortality rates ranging from $6.2 \%$ to $27 \%{ }^{1,2}$ Although multimodal and multidisciplinary perioperative care strategies may reduce postoperative mortality, ${ }^{2}$ safe and effective surgical treatment is fundamental to achieve favorable outcomes.
Because the lifetime prevalence of perforation in peptic ulcer patients is as high as $5 \%,{ }^{3}$ many surgical treatment modalities for the condition have been reported. Since laparoscopic repair procedures for perforated peptic ulcers were first reported in $1990,{ }^{4,5}$ laparoscopic surgical strategies have been described in many comparative 2005 suggested that patients with no surgical risk factors benefit from laparoscopic repair. ${ }^{6,7}$ Recent systematic reviews did not show significant differences 
between laparoscopic and open surgery in terms of abdominal septic and pulmonary complications, ${ }^{8}$ and results for laparoscopic surgery were comparable with those of open surgery in perforated peptic ulcer while also offering the advantages of minimally invasive treatment. ${ }^{9}$

However, there are concerns associated with laparoscopic repair of perforated peptic ulcer: (1) a higher leakage rate of $3.8 \sim 16 \%$ than with conventional treatments, ${ }^{6,7,10-13}$ (2) longer operation time, ${ }^{7,11,12}$ and (3) open conversion required in $12.4 \%$ of patients. ${ }^{13}$ Higher incidence of leakage at repaired perforation sites might be explained because surgeons avoid omental patches to shorten the operation time. ${ }^{13}$ Longer operation time is a pitfall of laparoscopic surgery in critically ill patients, and may be caused by difficulty in multiple laparoscopic suturing or additional omentopexy. Finally, larger perforations may require open conversion. ${ }^{14}$ while simple suturing without omental patching may lead to sutures tearing out and enlargement of the perforation due to injury to the friable tissue in the margins of the perforation. Therefore, we developed a novel laparoscopic technique that is easy, safe, and effective for omental patch repair in cases of perforated duodenal ulcer.

Laparoscopic repair for perforated peptic ulcers should be preferentially considered in patients without contraindications, if an experienced laparoscopic surgeon is available. Herein, we present our surgical technique for perforated duodenal ulcers, which we call laparoscopic single figure of eight suturing omentopexy, and its associated clinical outcomes. This is the first presentation of a formulaic surgical technique for a novel laparoscopic omentopexy.
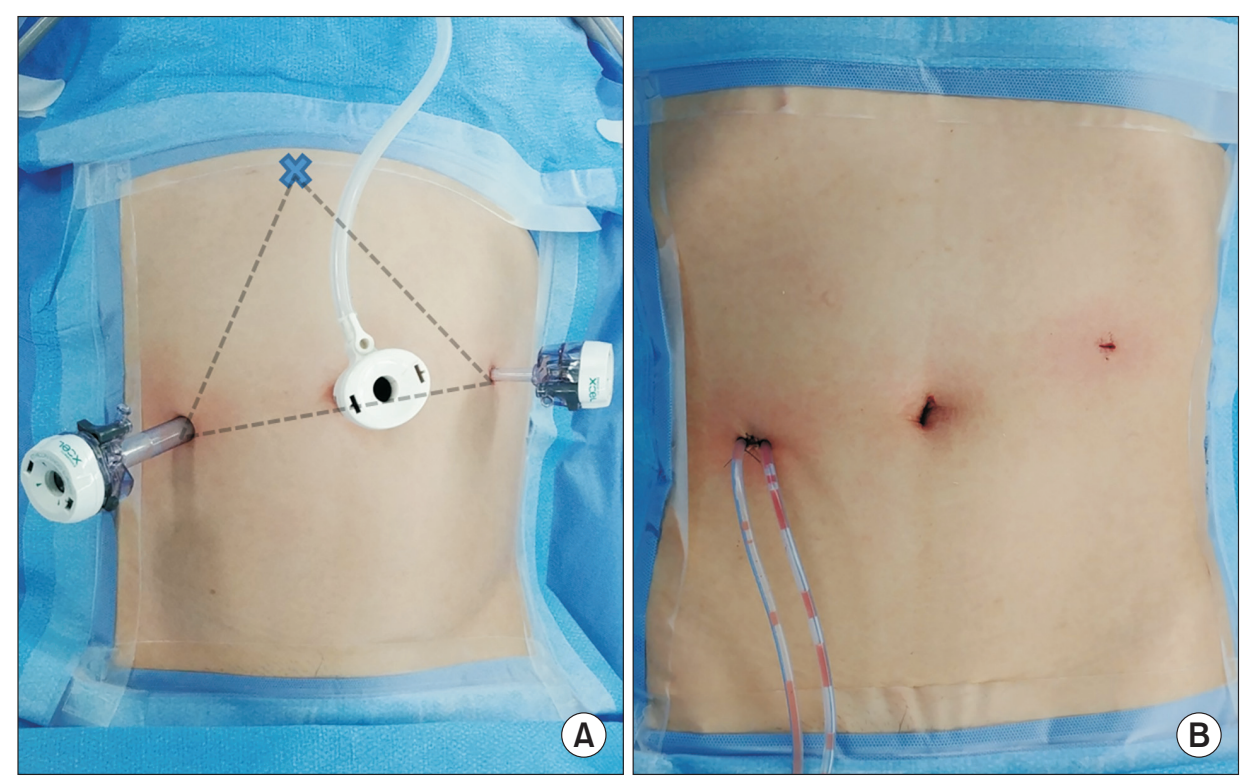

Fig. 1. Preparations for laparoscopic repair of a perforated duodenal ulcer in a 45-year old male patient. Alignment between the trocars is important to ease laparoscopic figure of eight suturing and tying for the omental patch. After trocar insertion in the form of an equilateral triangle directed to the operation site (A) and immediate postoperative wound and drain (B). 
trocar was inserted into the right mid- to lower abdomen under laparoscopic direct vision. The other $5 \mathrm{~mm}$ trocar was inserted into the left mid- to upper abdomen. The three trocars were inserted in the form of an equilateral triangle directed to the operation site (duodenal bulb). Adequate positioning was obtained with the right tilted upward in the Trendelenburg or anti-Trendelenburg position. Intraperitoneal exploration was performed to find the perforation site. Once the perforation site was found, laparoscopic figure of eight suturing omental patch was performed using 3-0 Coated VICRYL Plus Antibacterial (Ethicon, Inc., Somerville, NJ, USA) as follows (Fig. 2): (1) the needle was passed into the proximal duodenum to the ulcer some distance away from the margin of the perforation and brought out through the perforation, (2) the needle was reintroduced through the perforation and brought out through the wall of the distal duodenum to ulcer, (3) the needle was passed again below the point of the first needle passing and brought out through the perforation, (4) the needle was reintroduced through the perforation and brought out through the duodenal wall below the point of the previous needle passing at distal duodenum to ulcer, and (5) the pedicled omentum from a mobile portion of the greater omentum was introduced between the suture thread and perforated duodenal ulcer, and then intracorporeal laparoscopic tying of figure of eight suture was performed to complete omentopexy. After peritoneal toilet, two lines of closed suction drain were inserted into the subhepatic space through the right $12 \mathrm{~mm}$ trocar site (Fig. 2).

\section{Perioperative management}

Abdominal CT scans were taken in all patients in whom peptic ulcer perforation was suspicious. All patients received intravenous antibiotics (third-generation cephalosporin and metronidazole) after diagnosis. A urinary catheter and nasogastric tube were inserted in the emergency room. All anesthetic procedures were performed in routine fashion. Intravenous patient-controlled analgesia was provided for postoperative pain relief according to patient preference. The urinary catheter was removed at 6 am on the first postoperative day, after which all patients were encouraged to ambulate. Suction drainage via nasogastric tube continued after surgery. Upper gastrointestinal (GI) series using gastrografin were performed during the 3 5 days after surgery according to the availability of the radiologist. After confirmation that there was no leakage or obstruction from the operation site in the upper GI series, the nasogastric tube was removed. A clear liquid diet was started after bowel sounds were detected and signs of peritonitis improved, and then each patient was advanced to a regular diet when the full liquid diet was tolerated or flatus observed. Patients were discharged from the hospital to their homes after they were demonstrated to tolerate a regular diet and experienced normal bowel movement.
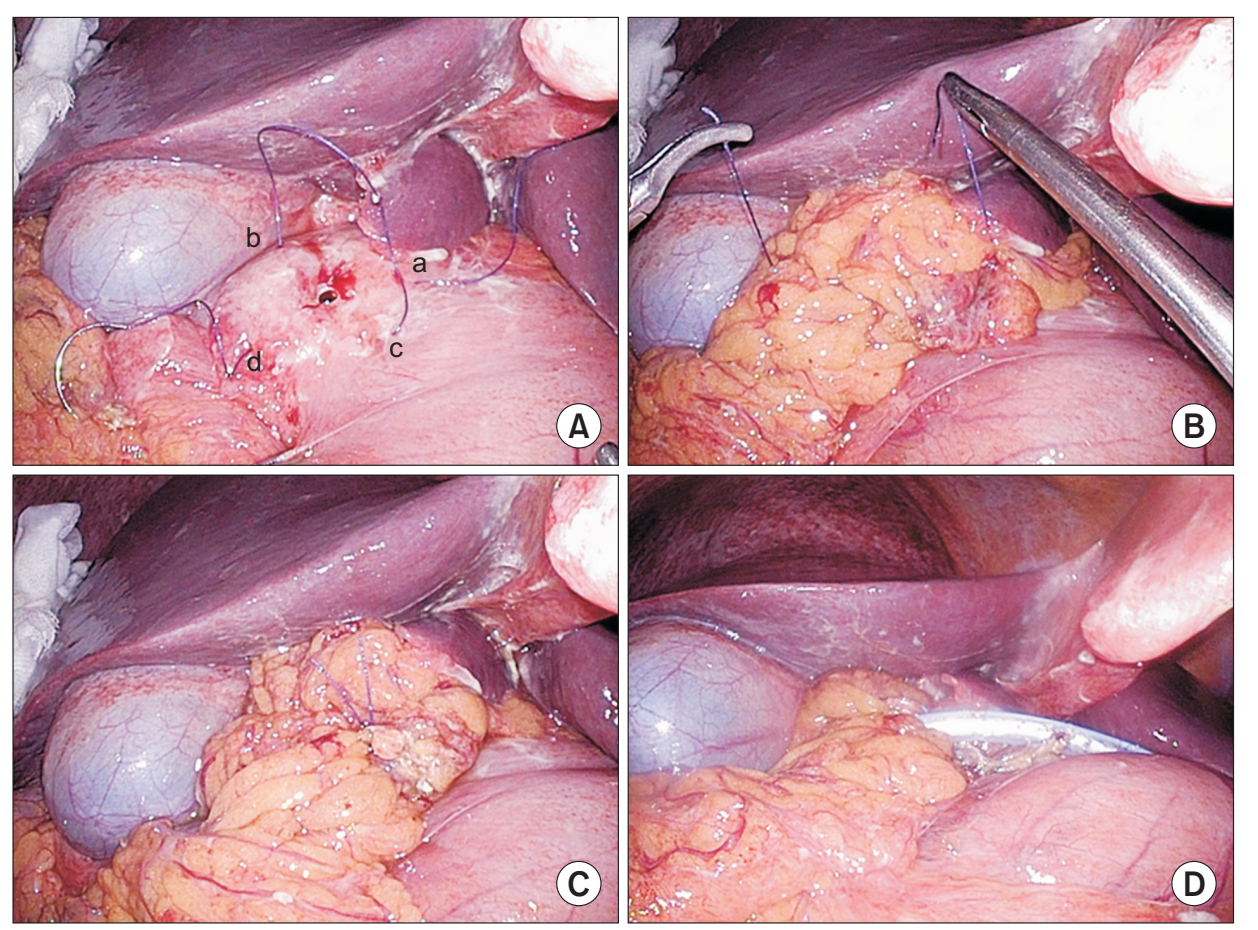

Fig. 2. Figure of eight suturing around a perforated duodenal ulcer, in the order of $a, b, c$, and d (A). Pedicled omentum from the mobile portion of the greater omentum was introduced between the suture thread and perforated duodenal ulcer $(B)$, and then intracorporeal laparoscopic tying of the figure of eight suture was performed to complete the omentopexy (C). Two lines of drains inserted into the subhepatic space on the repair site (D). 
Table 1. Clinical characteristics and operative findings

\begin{tabular}{lc}
\hline & Number of patients $(\mathbf{n}=15)$ \\
\hline Age (years) & $41.7 \pm 13.8$ \\
Gender (males/females) & $15 / 0$ \\
ASA score (\%) & $9(60.0)$ \\
1 & $6(40.0)$ \\
2 & $0(0)$ \\
$3 / 4$ & $2(13.3)$ \\
History of previous abdominal surgery (\%) & $11.2 \pm 5.5$ \\
Duration of perforation (hours) & $10(4.5 \sim 27.0)$ \\
& \\
Site of perforation (\%) & $15(100)$ \\
Duodenal bulb & $5.3 \pm 1.7$ \\
Size of perforation (mm) & $5(3 \sim 8)$ \\
\hline
\end{tabular}

Values are presented as the number $(\%)$ and the mean value \pm standard deviation or median value (range). ASA = American Society of Anesthesiologists.

\section{RESULTS}

\section{Patient characteristics}

A total of 15 patients with a mean age of $41.7 \pm 13.9$ years were included in the study sample. Clinical data including gender, ASA score, site and size of perforation and duration of perforation are shown in Table 1. Categorical data are presented as percentages, and quantitative data as the mean \pm standard deviation or median value with range.

\section{Perioperative outcomes}

All 15 patients underwent laparoscopic single figure of eight suturing omentopexy for the management of perforated duodenal ulcers. The most delayed patients underwent surgery 27 hours after symptoms occurred. Boey scores were 0 in all patients except for one for whom surgery was delayed. The perforation site was the anterior duodenal bulb in all patients. The median perforation size was $5 \mathrm{~mm}$ (range: $3 \sim 8 \mathrm{~mm}$ ). The mean operation time was $66.7 \pm 19.6$ minutes. There was no evidence of leakage from the omentopexy site clinically or in postoperative upper GI series in any of the patients. One patient $(6.7 \%)$ experienced the postoperative complication of pneumothorax in the right lung and atelectasis in both lungs. Postoperative pneumothorax occurred 3 days after surgery, which was initially managed by supportive treatment, such
Table 2. Perioperative outcomes and postoperative recovery

\begin{tabular}{lc}
\hline & Number of patients $(\mathbf{n}=15)$ \\
\hline Operation time $(\mathrm{min})$ & $66.7 \pm 19.6$ \\
& $60(40 \sim 105)$ \\
\hline Postoperative complications $(\%)$ & $1(6.7)$ \\
Pneumothorax & $1(6.7)$ \\
Reoperation within 30 days of surgery & $0(0)$ \\
30-day postoperative mortality & $0(0)$ \\
\hline Time to tolerance of regular diet (days) & $6(4 \sim 9)$ \\
\hline Postoperative hospital stay (days) & $7(5 \sim 11)$ \\
\hline
\end{tabular}

Values are presented as the number $(\%)$ and the mean value \pm standard deviation or median value (range).

as $\mathrm{O}^{2}$ administration at a high flow rate. However, the pneumothorax did not resolve spontaneously and dyspnea was aggravated, so a chest tube was inserted 5 days after surgery and removed 10 days after surgery. The patient was discharged from hospital to home 11 days after surgery. There were no cases of postoperative mortality or reoperation within 30 days after surgery. The median time to tolerance of regular diet was 6 days (range: 4 9 days). Median postoperative hospital stay was 7 days (range: $5 \sim 11$ days) (Table 2).

\section{DISCUSSION}

Laparoscopic surgery for the management of perforated peptic ulcers has advantages associated with minimally invasive surgery, such as lower surgical site infection, shorter hospital stays, and less postoperative pain. ${ }^{6,9,13}$ General surgical outcomes after laparoscopic surgery for management of perforated peptic ulcer, including overall postoperative complication rate, mortality, and reoperation rate, are comparable with those of open surgery. ${ }^{9}$ Laparoscopic management may be the treatment of choice, especially in patients without surgical risk factors such as elderly age, shock, symptoms persisting longer than 24 hours, or underlying medical illness, and should be considered first among surgical options for the management of perforated peptic ulcers. ${ }^{7,13}$

However, concerns remain regarding the laparoscopic treatment of perforated peptic ulcers. The incidence of leakage at the repair site may be higher than that of open surgery, and the reported leakage rates after laparoscopic repair range from $3.8 \sim 16 \%{ }^{67,10-13}$ In particular, the leakage rate after laparoscopic sutureless fibrin glue repair was reported to be $16 \%$, and therefore Lee et al. recommended that this method should be considered only in selected patients with low surgical risk and Acute Physiology And Chronic Health Evaluation (APACHE) 
II scores below $5{ }^{10}$ Large size of peptic ulcer perforation may also increase the rate of leakage after laparoscopic repair. ${ }^{10,14}$ Large ulcers are difficult to close using laparoscopic simple suturing not only due to the size of the defect, but due to the marked induration at the margin of the perforated ulcer. Simple suture and knot tying to approximate a defect with a marked induration by ulcer scar tissue tends to result in cutting through the margin of the perforation by the suture thread, which widens the defect. In such cases, the use of an omental patch plugged into the perforation instead of direct suture closure of the perforated ulcer could prevent traumatic injury. The use of a pedicled omental patch plugged into the perforation has been considered the gold standard since Cellan-Jones first presented this approach for management of perforated duodenal ulcer. ${ }^{15}$ The surgical technique described in this study is a simplified and modified version of the pedicled omental patch method plugged into the perforation for laparoscopic surgery.

Few studies comparing simple closure and omental patching for the laparoscopic repair of perforated peptic ulcer have been performed. These studies showed that laparoscopic simple closure is a safe procedure that shortens operation time, but were retrospective and non-randomized, and most enrolled patients did not have risk factors such as shock, long-standing perforation, generalized peritonitis, or high Boey scores. ${ }^{16,17}$ Prospective randomized studies should be performed to confirm the safety and effectiveness of laparoscopic simple suture approaches for treating perforated ulcers. Indeed, laparoscopic omental patches may require additional technical skill and are time consuming, so laparoscopic repair of perforated peptic ulcer without omental patching could shorten operation time relative to that required for open repair. ${ }^{18}$ However, our single figure of eight suturing omentopexy method could resolve problems associated with laparoscopy such as longer operation time or higher technical skill requirements, thus obviating the necessity of prospective randomized studies. Using our techniques, the insertion of an omental plug into the perforation could both simplify the procedure and shorten operation time. In the present study, the mean operation time was $66.7 \pm 19.6$ minutes, which is comparable to previously reported results for laparoscopic simple closure techniques without omental patch. $^{16,17}$

Open conversion rates have been reported to range from $0 \%$ to $28.5 \%$, and the most common causes of conversion are large perforation size (usually $>10 \mathrm{~mm}$ ), inadequate ulcer location, and difficult suturing due to friable ulcer edge. ${ }^{13}$ In the present study, large $(>10 \mathrm{~mm})$ duodenal wall defects as visualized on perioperative CT scan were treated by open surgery and $\mathrm{ex}^{-}$ cluded from the study, so it is impossible to determine whether larger duodenal ulcer perforations can be safely managed us- ing our surgical technique. However, omental patching is a safe method that has been widely used in surgical approaches for the treatment of large perforated duodenal ulcer for nine decades, after Cellan-Jones first reported the use of the pedicled omental patch in $1929 .{ }^{15}$ Therefore, given the favorable surgical outcomes observed at our institution, we are currently trying to extend our inclusion criteria for laparoscopic repair of perforated duodenal ulcer to include ulcers larger than 10 $\mathrm{mm}$ in perforation size.

Perforated duodenal ulcers in the anterior and duodenal first portion are usually easily located using laparoscopy. However, gastric ulcers or those with posterior localization may be difficult to locate and manage by laparoscopy, and therefore we believe such ulcers should be managed by open surgery or definitive ulcer surgery rather than laparoscopic closure of the perforation. We refer patients suspicious of inadequate ulcer localization to surgeons specializing in upper GI (especially gastroduodenal) surgery, according to our institutional protocol. In the present study, 15 consecutive laparoscopic repairs of perforated duodenal ulcers were performed by one of three experienced laparoscopic surgeons (two colon and rectal surgeons and one hepatobiliary and pancreatic surgeon) because they were included among the surgical on-call team at our institute. Our surgical technique could easily be performed by any experienced laparoscopic surgeon, regardless of subspecialty in general surgery.

Another common cause of open conversion, difficult suturing due to friable ulcer edges, also may be overcome by figure of eight suturing. ${ }^{19}$ Figure of eight suturing could be used to avoid cutting through the margin of the perforation by the suture thread, since the pressure that would be aligned to a single axis of a simple interrupted suture is applied to two axes in figure of eight sutures. Furthermore, our technique of omentopexy is different from omental patch techniques using uncut suture thread on top of the perforation closed by simple suture and knot tying. Figure of eight suturing and omental patch application plugged into the perforation could create a synergistic effect resulting in avoidance of iatrogenic traumatic injury at friable ulcer margins.

Spontaneous pneumothorax occurred in one patient 3 days after surgery. This patient had the longest postoperative hospital stay (11 days) among those included in the present study. Postoperative pneumothorax following laparoscopic surgery is a rare complication that occurs in only $0.24 \%$ of patients. ${ }^{20}$ Spontaneous pneumothorax might not be related to surgery, but prolonged operation time and increased intra-abdominal pressure are possible contributing factors. ${ }^{21}$ Early diagnosis of postoperative pneumothorax using chest $\mathrm{X}$-ray and prompt management, including supportive treatment with $\mathrm{O}^{2}$ administration at a high flow rate and chest tube insertion if neces- 
sary, are critical. In the present study, there were no cases of leakage at the repair site or abdominal septic complication following laparoscopic single figure of eight suturing omentopexy.

In conclusion, laparoscopic repair should be considered first for the management of perforated duodenal ulcers in selected patients without surgical risk factors, if there is an experienced laparoscopic surgeon on hand. Laparoscopic single figure of eight suturing omentopexy can be a viable option in the treatment of choice for the surgical management of perforated duodenal ulcers, and appears to be a safe, easy, and effective surgical technique that shortens operation time.

\section{REFERENCES}

1) Boey J, Choi SK, Poon A, Alagaratnam TT. Risk stratification in perforated duodenal ulcers. A prospective validation of predictive factors. Ann Surg 1987;205:22-26.

2) Moller MH, Adamsen S, Thomsen RW, Moller AM. Multicentre trial of a perioperative protocol to reduce mortality in patients with peptic ulcer perforation. Br J Surg 2011;98:802-810.

3) Vaira D, Menegatti M, Miglioli M. What is the role of Helicobacter pylori in complicated ulcer disease? Gastroenterology 1997;113: S78-84.

4) Mouret P, Francois Y, Vignal J, Barth X, Lombard-Platet R. Laparoscopic treatment of perforated peptic ulcer. Br J Surg 1990;77: 1006.

5) Nathanson LK, Easter DW, Cuschieri A. Laparoscopic repair/ peritoneal toilet of perforated duodenal ulcer. Surg Endosc 1990;4: 232-233.

6) Lunevicius R, Morkevicius M. Systematic review comparing laparoscopic and open repair for perforated peptic ulcer. Br J Surg 2005;92:1195-1207.

7) Lunevicius R, Morkevicius M. Management strategies, early results, benefits, and risk factors of laparoscopic repair of perforated peptic ulcer. World J Surg 2005;29:1299-1310.

8) Sanabria A, Villegas MI, Morales Uribe CH. Laparoscopic repair for perforated peptic ulcer disease. Cochrane Database Syst Rev 2013:Cd004778.
9) Tan S, Wu G, Zhuang Q, et al. Laparoscopic versus open repair for perforated peptic ulcer: A meta analysis of randomized controlled trials. Int J Surg 2016;33 Pt A:124-132.

10) Lee FY, Leung KL, Lai PB, Lau JW. Selection of patients for laparoscopic repair of perforated peptic ulcer. Br J Surg 2001;88:133136.

11) Lunevicius R, Morkevicius M. Comparison of laparoscopic versus open repair for perforated duodenal ulcers. Surg Endosc 2005;19: 1565-1571.

12) Bertleff MJ, Halm JA, Bemelman WA, et al. Randomized clinical trial of laparoscopic versus open repair of the perforated peptic ulcer: the LAMA Trial. World J Surg 2009;33:1368-1373.

13) Bertleff MJ, Lange JF. Laparoscopic correction of perforated peptic ulcer: first choice? A review of literature. Surg Endosc 2010; 24:1231-1239.

14) Kim JH, Chin HM, Bae YJ, Jun KH. Risk factors associated with conversion of laparoscopic simple closure in perforated duodenal ulcer. Int J Surg 2015;15:40-44.

15) Cellan-Jones CJ. A rapid method of treatment in perforated duodenal ulcer. Br Med J 1929;1:1076-1077.

16) Abd Ellatif ME, Salama AF, Elezaby AF, et al. Laparoscopic repair of perforated peptic ulcer: patch versus simple closure. Int J Surg 2013;11:948-951.

17) Lin BC, Liao CH, Wang SY, Hwang TL. Laparoscopic repair of perforated peptic ulcer: simple closure versus omentopexy. J Surg Res 2017;220:341-345.

18) Ates M, Sevil S, Bakircioglu E, Colak C. Laparoscopic repair of peptic ulcer perforation without omental patch versus conventional open repair. J Laparoendosc Adv Surg Tech A 2007;17:615619.

19) Gupta SP. A safer technique of closure of peptic ulcer perforation. Indian J Surg 2011;73:361-362.

20) Graybill WS, Frumovitz M, Nick AM, et al. Impact of smoking on perioperative pulmonary and upper respiratory complications after laparoscopic gynecologic surgery. Gynecol Oncol 2012;125:556560.

21) Paul PG, Mathew $T$, Shintre $H$, Bulusu $S$, Paul G, Mannur $S$. Postoperative Pulmonary Complications Following Laparoscopy. J Minim Invasive Gynecol 2017;24:1096-1103. 